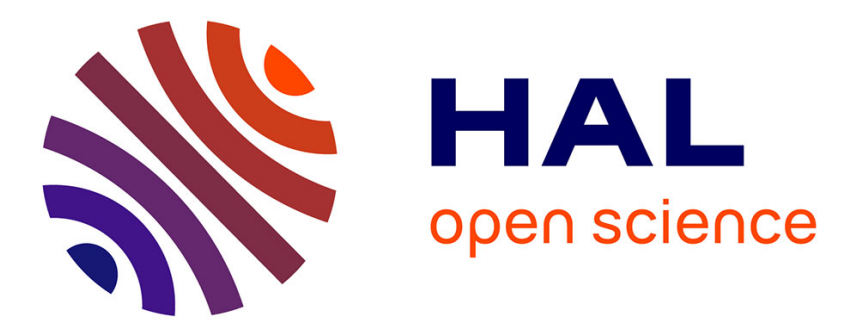

\title{
Event-triggered attitude control for flying robots using an event approach based on the control
}

\author{
Jose Fermi Guerrero Castellanos, Nicolas Marchand, Sylvain Durand, A
}

Vega-Alonzo, Jose Juan Téllez-Guzmán

\section{- To cite this version:}

Jose Fermi Guerrero Castellanos, Nicolas Marchand, Sylvain Durand, A Vega-Alonzo, Jose Juan Téllez-Guzmán. Event-triggered attitude control for flying robots using an event approach based on the control. EBCCSP 2015 - 1st IEEE Conference on Event-Based Control Communication and Signal Processing, Jun 2015, Krakow, Poland. hal-01171459

\section{HAL Id: hal-01171459 \\ https://hal.science/hal-01171459}

Submitted on 3 Jul 2015

HAL is a multi-disciplinary open access archive for the deposit and dissemination of scientific research documents, whether they are published or not. The documents may come from teaching and research institutions in France or abroad, or from public or private research centers.
L'archive ouverte pluridisciplinaire HAL, est destinée au dépôt et à la diffusion de documents scientifiques de niveau recherche, publiés ou non, émanant des établissements d'enseignement et de recherche français ou étrangers, des laboratoires publics ou privés. 


\title{
Event-triggered attitude control for flying robots using an event approach based on the control
}

\author{
J.F. Guerrero-Castellanos, N. Marchand, S. Durand, A. Vega-Alonzo, J.J. Téllez-Guzmán
}

\begin{abstract}
This paper presents the development of a quaternion-based nonlinear event-triggered control for the attitude stabilization of Flying robots. Firstly, it is proved the existence of a Control Lyapunov Function. Unlike some previously proposed schemes, the aim of this paper is to propose a new and simpler event function. The control law ensures the asymptotic stability of the closed-loop system to the desired attitude. The approach is validated in real-time using a quadrotor mini-helicopter. The experiments show that the event driven controller reduces the control update without deteriorating the closed-loop system performance.
\end{abstract}

\section{INTRODUCTION}

Flying robots and Unmanned Aerial Vehicles (UAVs) have received growing interest in industrial and academic research. They may prove useful for many civilian missions. Furthermore, among miniature rotorcraft-based UAVs, the mini quadrotor helicopter gives rise to great interest because of its high manoeuvrability, its payload capacity and its ability to hover, as explained in [1]. Such a Vertical Take-Off and Landing (VTOL) vehicle has some advantages over conventional helicopters: owing to symmetry, it is relatively simple to design and construct. In fact, the quadrotor is an under-actuated dynamic system with four input forces and six output coordinates (attitude and position). However, this system can be broken down into two subsystems, one defining the translation movement and the other one the rotation movement. These subsystems are coupled in cascade since the translational subsystem depends on the rotational one, but the rotational subsystem is independent of the translational one. Self-governing flights require the generation of low-level control signals sent to actuators as well as decision-making related to guidance, navigation. Low-level flight control is known as attitude control and it is responsible for maintaining the desired vehicle orientation. Consequently, the attitude controller design is, in itself, a challenge.

Some linear and nonlinear control techniques have been applied for the attitude stabilization of the quadrotor, like for example in [2], [3], [4], [5], [6], [7], [8].

This work was partially supported by VIEP-BUAP under grant GUCJING15-I

J.F. Guerrero-Castellanos and A. Vega-Alonzo are with Autonomous University of Puebla (BUAP), Faculty of Electronics, Puebla, Mexico, \{fguerrero, aalonzo\}@ece.buap.mx

${ }^{4}$ S. Durand is with Aix-Marseille University, ISM, FR-13009, Marseille, France. sylvainedurandchamontin. fr

N. Marchand and J.J. Téllez-Guzmán is with GIPSA-lab laboratory, Control Systems Dept., SySCo team, CNRS-Univ. of Grenoble, ENSE3 BP 46, 38402 St Martin d'Hères Cedex, France, nicolas.marchand, jose-juan.tellez-guzman@gipsa-lab.inpg.fr
Actually, all proposed attitude control laws previously listed were developed in continuous time framework and their implementation under digital platforms is carried out by means of "emulation". This procedure consists in implementing a continuous time control algorithm with a constant and sufficiently small periodic sampling period. However, this approach can be constrained by hardware and reducing the sampling period to a level that guarantees acceptable closed-loop performance may be impossible.

On the other hand, in the recent years, some works addressed resource-aware implementations of the control law using event-triggered sampling, where the control value is updated only when some events occur. An event is usually generated by an event-function that indicates if the control signal must be updated or not. Typical event-detection mechanisms are functions on the variation of the state (or at least the output) of the system, like in [9], [10], [11], [12], [13], [14]. In [15] in particular, it is proved that such an approach reduces the number of sampling instants for the same final performance. An event-triggered paradigm hence calls for resources whenever they are indeed necessary. In the same idea, an alternative approach consists in taking events related to the variation of a Lyapunov function between the current state and its value at the last sampling, like in [16], or in taking events related to the time derivative of the Control Lyapunov Function (CLFs), like in [17], [18], [19]. In this latter case, the updates ensure the strict decrease of a Control Lyapunov function, and so is ensured the asymptotically stability of the closed-loop system.

Although the advantages of event-triggered control are well-motivated and theoretical results show its potential, few results in the framework of unmanned aircraft systems have been presented in literature, e.g. [20], [21]. In these works linear event-triggered controllers are proposed for attitude stabilization of a 3D helicopter model. Unfortunately, these controllers only work in a limited attraction region of the state-space.

In the present work, we developed an event-triggered non linear control strategy for the attitude stabilization of Flying Robots. The feedback is quaternion-based and it is derived from the universal formula for event-triggered stabilization of general nonlinear systems affine in the control [18] but using an event function based in the control as proposed in [19] . The proposed feedback ensures the asymptotic stability and it is smooth everywhere and continuous at the origin. Moreover, we propose to test such a proposal on a real-time system, namely, a quadrotor mini-helicopter. The idea is to show that an event-triggered scheme could reduce 
the number of samples even in such a case where rotor blades have to be actively controlled.

The paper is organized as follows. First, in section II we present some mathematical definitions and the event-based control strategy for affine in the control nonlinear systems is detailed. The quaternion notion is also introduced. The section III states the problem and presents the design of the control law for the attitude stabilization. The quadrotor mini-helicopter model is given in section IV where experimental results are presented and discussed. Some reflections conclude the paper.

\section{PRELIMINARIES}

In this section some facts for event-triggered stabilization of general nonlinear systems affine in the control [18] are reviewed and the system model is introduced [7].

A. Event-triggered stabilization for affine in the control dynamical systems

In this paper, the study will focus on affine in the control dynamical systems defined by:

$$
\dot{x}=f(x)+g(x) u
$$

where $x \in \mathcal{X} \subset \mathbb{R}^{n}, u \in \mathcal{U} \subset \mathbb{R}^{p}$, and $f$ a Lipschitz function vanishing at the origin. For the sake of simplicity, we only consider in this paper null stabilization with initial time instant $t_{0}=0$. If the system (1) admits an asymptotic stabilizing feedback $k: \mathcal{X} \rightarrow \mathcal{U}$ then there exists a Control Lyapunov Function (CLF) $V: \mathcal{X} \rightarrow \mathbb{R}$, that is a smooth function, positive definite and such that:

$$
\dot{V}=\underbrace{\frac{\partial V^{T}}{\partial x} f(x)}_{:=a(x)}+\underbrace{\frac{\partial V^{T}}{\partial x} g(x)}_{:=b(x)} k(x)<0
$$

It is worth noting that if $k$ is assumed to be smooth, then $V$ is known to exist and to be as smooth as $k$. In the present paper, only the smoothness of $V$ is required which is less restrictive than the one of $k$. An equivalent requirement on the time derivative of $V$ is that:

$$
b(x)=0 \Rightarrow a(x)<0
$$

Event-triggered feedback usually means a set of two functions:

- an event function $e: \mathcal{X} \times \mathcal{X} \rightarrow \mathbb{R}$ that indicates if one needs $(e \leq 0)$ or not $(e>0)$ to update the control value. Event function $e$ takes the current state $x$ as input and a memory $m$ of $x$ last time $e$ became negative.

- a feedback function $k: \mathcal{X} \rightarrow \mathcal{U}$. Which is used as in the classical frame.

We recall here the definition of semi-uniform Minimum Sampling Interval (MSI) event-triggered control:

Definition 2.1: [18] An event-triggered feedback $(k, e)$ is said to be semi-uniformly MSI if for all $\delta>0$, and all $x_{0}$ in the ball of radius $\delta$ centred at the origin $\mathcal{B}(\delta)$ the inter-execution times, that is the duration between two successive events, can be below bounded by some $\underline{\tau}>0$.
Remark 2.2: This minimal sampling period is useful for implementation purpose but also when the feedback $k$ is discontinuous for robustness purpose [22] as this one proposed in the present paper.

It is known that a nonlinear system of the form (1) with a semi-uniformly MSI event-based feedback $(e, k)$, the solution of (1) starting in $x_{0} \in \mathcal{X}$ at $t=0$ is defined for all positive $t$ as the solution of the differential system:

$$
\begin{aligned}
& \dot{x}=f(x)+g(x) k(m)) \\
& \left\{\begin{array}{c}
m=x \quad \text { if } e(x, m) \leq 0, x \neq 0 \\
\dot{m}=0 \quad \text { elsewhere }
\end{array}\right. \\
& \text { with } x(0):=x_{0} \text { and } m(0)=x(0)
\end{aligned}
$$

Theorem 2.3: (Event-Triggered universal formula with an event function based on the control) If there exists a CLF for system (1), then the event-based feedback $(e, k)$ defined below is semi-uniformly MSI, smooth on $\mathcal{X} \backslash\{0\}$, and such that:

$$
\frac{\partial V}{\partial x} f(x)+\frac{\partial V}{\partial x} g(x) k(m)<0, \quad x \in \mathcal{X} \backslash\{0\}
$$

where $m$ is defined in (4) and:

$$
k_{i}(x):=-b_{i}(x) \delta_{i}(x) \gamma(x)
$$

$e(x, m):= \begin{cases}\inf _{i \in\{1, \ldots, p\}} \bar{\theta}(x) \delta_{i}(x)-p\left[k_{i}(m)-k_{i}(x)\right]^{2} & \text { on } \mathcal{S} \\ 1 & \text { otherwise }\end{cases}$

where

- $a(x):=\frac{\partial V}{\partial x} f(x)$ and $b(x):=\frac{\partial V}{\partial x} g(x)$,

- $x \rightarrow \Delta(x):=\operatorname{diag}\left(\delta_{1}(x), \delta_{2}(x), \ldots, \delta_{p}(x)\right)$ is a smooth function of $\mathcal{X} \backslash\{0\}$ to $\mathbb{R}^{p \times p}$, positive definite on:

$$
\mathcal{S}:=\{x \in \mathcal{X} \mid\|b(x)\| \neq 0\}
$$

- $x \rightarrow \bar{\theta}(x)$ is a smooth positive function of $\mathcal{X}$ to $\mathbb{R}$, such that $\bar{\theta}(x)\|\Delta(x)\|$ vanishes at the origin, and ensuring on $\mathcal{S} \backslash\{0\}$ the inequality $a(x)^{2}+\bar{\theta}(x) b(x) \Delta(x) b(x)^{T}>0$ - $\gamma: \mathcal{X} \rightarrow \mathbb{R}$ is defined by:

$$
\gamma(x):= \begin{cases}\frac{a(x)+\sqrt{a(x)^{2}+\bar{\theta}(x) b(x) \Delta(x) b(x)^{T}}}{b(x) \Delta(x) b(x)^{T}} & \text { if } x \in \mathcal{S} \\ 0 & \text { if } x \notin \mathcal{S}\end{cases}
$$

The proof of Theorem 2.3 was given in [19]. However, here one would like to mention the advantages and properties of the control law (7) and the idea behind the construction of the event function (8).

First, the feedback (7) is based on the general formula for the stabilization of nonlinear systems proposed by E. Sontag in [23] in the continuous framework. In [18] and [19] we extended the results to the event-triggered framework and we showed that this control law is smooth everywhere and continuous at the origin. It is well known that, there do not exist systematic techniques for finding CLFs for general nonlinear systems, but the proposed approach can be applied successfully to many types of systems for which CLFs can 
be found. On the another hand, one would like to determine when it is indeed necessary to applied the control law in order to guarantee the closed-loop stability. To derive a stabilizing triggering rule, one looks at the discrepancy between the control that should be applied if in this moment there is an event, that is, $k(x)$ and the last applied control, i.e. $k(m)$. For this, one looks the time derivative of $V$ along the trajectories of the system using the control law (7):

$$
\begin{aligned}
\dot{V} & =a(x)+b(x) k(m) \\
& =a(x)+b(x) k(x)+b(x)(k(m)-k(x)) \\
& =-\sqrt{a(x)^{2}+\theta(x) b(x) \Delta(x) b(x)^{T}}+b(x)(k(m)-k(x))
\end{aligned}
$$

Note that right after an event occurs $k(m)=k(x)$ and the time derivative of $V$ becomes negative, which is evidently the desired behaviour. However, in inter-event period, stability of the closed loop system can be guaranteed if $|b(x)(k(m)-k(x))|<\sqrt{a(x)^{2}+\theta(x) b(x) \Delta(x) b(x)^{T}}$. Furthermore, in the case $b(x)=0$ the stability remains due to the definition of CLF and thanks to the small control property the control is continuous at the origin. Hence, closed-loop stability can be enforced by executing the control task whenever

$$
\sqrt{a(x)^{2}+\theta(x) b(x) \Delta(x) b(x)^{T}}>|b(x)(k(m)-k(x))|
$$

This last inequality can be further transformed:

$$
\theta(x) \delta_{i}(x)>p\left[k_{i}(m)-k_{i}(x)\right]^{2}
$$

where $i \in\{1, \ldots, p\}$. Since inequality (11) is computationally less consuming than (10), this last is used for the construction of the event function (8).

\section{B. Flying robots attitude kinematics and dynamics}

In practice, the body of the flying robots can be considered rigid. The attitude of the flying robots can be parametrized by many ways. These parametrizations can be Euclidean, as in the case of Euler angles, which lie in $\mathbb{R}^{3}$, or non-Euclidean, as in the case of rotation matrices or quaternions. In this work, the unit quaternion is used and the mathematical background is presented below.

Consider two orthogonal right-handed coordinate frames: the body coordinate frame, $\mathbf{E}^{b}=\left[\vec{e}_{1}^{b}, \vec{e}_{2}^{b}, \vec{e}_{3}{ }^{b}\right]$, located at the center of mass of the rigid body and the inertial coordinate frame, $\mathbf{E}^{f}=\left[\vec{e}_{1}^{f}, \vec{e}_{2}{ }^{f}, \vec{e}_{3}{ }^{f}\right]$, located at some point in the space. The rotation of the body frame $\mathbf{E}^{b}$ with respect to the fixed frame $\mathbf{E}^{f}$ is represented by the attitude matrix $R \in S O(3)=\left\{R \in \mathbb{R}^{3 \times 3}: R^{T} R=I\right.$, det $\left.R=1\right\}$.

The cross product between two vectors $\xi, \chi \in \mathbb{R}^{3}$ is represented by a matrix multiplication $\left[\xi^{\times}\right] \chi=\xi \times \chi$, where $\left[\xi^{\times}\right]$is the well known skew-symmetric matrix.

The $n$-dimensional unit sphere embedded in $\mathbb{R}^{n+1}$ is denoted as $\mathbb{S}^{n}=\left\{x \in \mathbb{R}^{n+1}: x^{T} x=1\right\}$. Members of $S O(3)$ are often parametrized in terms of a rotation $\beta \in \mathbb{R}$ about a fixed axis $e_{v} \in \mathbb{S}^{2}$ by the map $\mathcal{U}: \mathbb{R} \times \mathbb{S}^{2} \rightarrow S O(3)$ defined as

$$
\mathcal{U}\left(\beta, e_{v}\right):=I_{3}+\sin (\beta)\left[e_{v}^{\times}\right]+(1-\cos (\beta))\left[e_{v}^{\times}\right]^{2}
$$

Hence, a unit quaternion, $q \in \mathbb{S}^{3}$, is defined as

$$
q:=\left(\begin{array}{c}
\cos \frac{\beta}{2} \\
e_{v} \sin \frac{\beta}{2}
\end{array}\right)=\left(\begin{array}{c}
q_{0} \\
q_{v}
\end{array}\right) \in \mathbb{S}^{3}
$$

$q_{v}=\left(\begin{array}{lll}q_{1} & q_{2} & q_{3}\end{array}\right)^{T} \in \mathbb{R}^{3}$ and $q_{0} \in \mathbb{R}$ are known as the vector and scalar parts of the quaternion respectively. $q$ represents an element of $S O(3)$ through the map $\mathcal{R}: \mathbb{S}^{3} \rightarrow S O(3)$ defined as

$$
\mathcal{R}:=I_{3}+2 q_{0}\left[q_{v}^{\times}\right]+2\left[q_{v}^{\times}\right]^{2}
$$

Note that $R=\mathcal{R}(q)=\mathcal{R}(-q)$ for each $q \in \mathbb{S}^{3}$, i.e. quaternions $q$ and $-q$ represent the same physical attitude. These two postures differ mathematically by a $2 \pi$ rotation about the fixed axis $e_{v}$.

Denoting by $\omega=\left(\begin{array}{lll}\omega_{1} & \omega_{2} & \omega_{3}\end{array}\right)^{T}$ the angular velocity vector of the body coordinate frame, $\mathbf{E}^{b}$ relative to the inertial coordinate frame, $\mathbf{E}^{f}$, expressed in $\mathbf{E}^{b}$, the complete attitude motion of a flying robot can be described by the state equations:

$$
\begin{aligned}
\dot{q} & =\frac{1}{2} \Xi(q) \omega \\
J \dot{\omega} & =-\left[\omega^{\times}\right] J \omega+\Gamma
\end{aligned}
$$

where

$$
\Xi(q)=\left(\begin{array}{c}
-q_{v}^{T} \\
I_{3} q_{0}+\left[q_{v}^{\times}\right]
\end{array}\right)
$$

$J$ is the inertia matrix, which is positive definite, and $\Gamma \in \mathbb{R}^{3}$ is the applied control input.

The attitude error is used to quantify the mismatch between two attitudes. If $q$ defines the current attitude quaternion and $q_{d}$ is the desired quaternion, i.e. the desired orientation, then the error quaternion that represents the attitude error between the current orientation and the desired one is given by

$$
q_{e}=q_{d}^{-1} \otimes q=\left(q_{e_{0}} q_{e_{v}}^{T}\right)^{T}
$$

where $q^{-1}$ is the complementary rotation of the quaternion $q$ which is given by $q^{-1}=\left(\begin{array}{ll}q_{0} & -q_{v}^{T}\end{array}\right)^{T}$ and $\otimes$ denotes the quaternion multiplication [24]. In the case that the current quaternion and the desired one coincide, the quaternion error becomes $q_{e}=\left( \pm 10^{T}\right)^{T}$.

As it was mentioned before, the quaternion representation is redundant. As a consequence, the error mathematical model has two equilibrium and this fact must be considered in the stability analysis [25].

\section{EVENT-BASED CONTROL STRATEGY}

\section{A. Problem statement}

The objective is to design a event-triggered control law that drives the flying robot attitude to a specified constant attitude starting from any initial condition. It follows that the angular velocity vector must be approach zero and remains null. In this paper, null stabilization is considered. Hence, 
the inertial coordinate frame is selected to be the desired orientation and the control objective is described by the following asymptotic condition:

$$
q \rightarrow\left( \pm 1 \quad 0^{T}\right)^{T}, \quad \omega \rightarrow 0 \text { as } t \rightarrow \infty
$$

Equation (18) represents two equilibrium points $\left(q_{0}=1\right.$, $\left.q_{v}=\left(\begin{array}{lll}0 & 0 & 0\end{array}\right)^{T}\right)$ and $\left(q_{0}=-1, q_{v}=\left(\begin{array}{lll}0 & 0 & 0\end{array}\right)^{T}\right)$. These equilibrium points represent the same equilibrium point in the physical space and they yield the same attitude matrix in equation (14). However, they represent two-point set in $\mathbb{S}^{3}$. This topological obstruction does not allows to state any global property for the closed-loop system, using a continuous quaternion-based feedback [26], [27].

In this study, the case $q_{d}=\left(\begin{array}{ll}1 & 0^{T}\end{array}\right)^{T}$ is considered.

On the other hand, it is well known that the flying robots are in general equipped of an Attitude Heading Reference Systems (AHRS) which provides 3D orientation by integrating gyroscopes and fusing this data with accelerometer and magnetometer data via Kalman filters or nonlinear observers (see Fig. 1). The AHRS continuously monitors the state $x$ (attitude and angular velocity). Based on current state information and the last computed control signal, which is piecewise constant, the event-function decides when to broadcast the current state measurement over the network which is denoted by $x_{i}$. Whenever the control block receives a new state value, it updates the control law and the control signal for the actuators. Then, it broadcasts the control signal over the network in order to evaluate the event-triggered function and to detect a new event.

Thus, the problem consists in showing that the attitude of the flying robot can be asymptotically stabilized by means of an event-triggered feedback as defined in section II-A, i.e. with the control law (7) together with the event function (8). Another motivation is that other traffic exists between two successive events and after the update and broadcasting of the control signal over the network. Reducing the traffic used for control (thanks to an event-based approach) hence allows i) to reduce traffic congestion in the network and ii) to broadcast other sensors data, for instance GPS, infrared sensors, laser range finder or vision.

\section{B. Control design}

Defining the variables $x_{1}=q_{0} \in \mathbb{R}, x_{2}=q_{v} \in \mathbb{R}^{3}$, $x_{3}=\omega \in \mathbb{R}^{3}$, (15) can be rewritten as

$$
\begin{aligned}
\dot{x}_{1} & =-\frac{1}{2} x_{2}^{T} x_{3} \\
\dot{x}_{2} & =\frac{1}{2}\left(x_{1} I_{3}+\left[x_{2}^{\times}\right]\right) x_{3} \\
\dot{x}_{3} & =-J^{-1}\left[x_{3}^{\times}\right] J x_{3}+J^{-1} u
\end{aligned}
$$

Actually, (19)-(21) have the following form

$$
\dot{x}=f(x)+g(x) u
$$

which is a nonlinear system affine in the control with state $x=\left(\begin{array}{llll}x_{1} & x_{2}^{T} & x_{3}^{T}\end{array}\right)^{T}$ and vectors fields

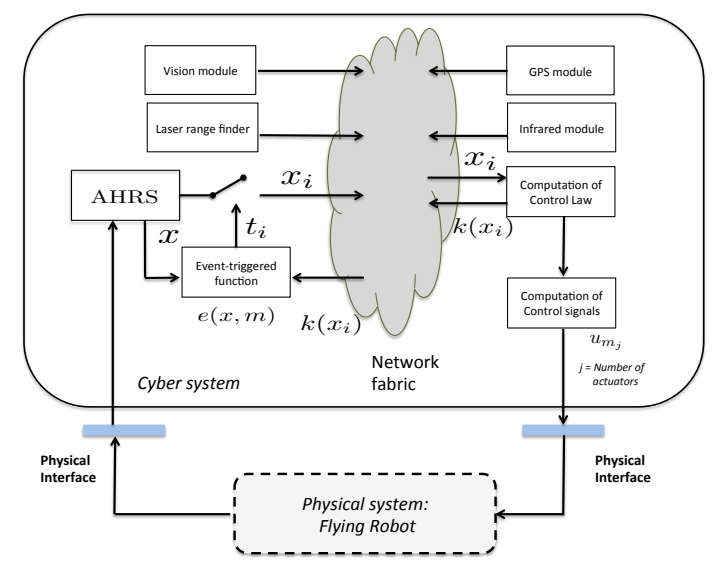

Fig. 1. Quadrotor control system

$$
\begin{aligned}
& f(x)=\left(\begin{array}{c}
-\frac{1}{2} x_{2}^{T} x_{3} \\
\frac{1}{2}\left(x_{1} I_{3}+\left[x_{2}^{\times}\right]\right) x_{3} \\
-J^{-1}\left[x_{3}^{\times}\right] J x_{3}
\end{array}\right) \\
& g(x)=\left(g_{1}(x) g_{2}^{T}(x) g_{3}^{T}(x)\right)^{T}
\end{aligned}
$$

where $g_{1}(x)=0 \in \mathbb{R}^{1 \times 3}, g_{2}(x)=0 \in \mathbb{R}^{3 \times 3}$ and $g_{3}(x)=$ $J^{-1} \in \mathbb{R}^{3 \times 3}$.

As a consequence, the control objective becomes

$$
x_{1} \rightarrow 1, \quad x_{2}, x_{3} \rightarrow 0 \text { as } t \rightarrow \infty
$$

In order to obtain a Control Lyapunov Function (CLF) of the system, which is necessary for the design of the event-triggered feedback, the Backsteeping procedure is carried out.

Consider firstly the subsystem (19)-(20) with $x_{3}$ a virtual control and let the control law:

$$
x_{3}=-K_{1} x_{2}
$$

where $K_{1}=I_{3} k_{1}$ and $k_{1} \in \mathbb{R}^{+}$. With this control law, the closed control-loop system becomes:

$$
\begin{aligned}
\left(\begin{array}{c}
\dot{x}_{1} \\
\dot{x}_{2}
\end{array}\right) & =-\frac{1}{2} K_{1}\left(\begin{array}{c}
-x_{2}{ }^{T} \\
I_{3} x_{1}+\left[x_{2} \times\right]
\end{array}\right) x_{2} \\
& =-\frac{1}{2} K_{1} \Xi\left(x_{1}, x_{2}\right) x_{2}
\end{aligned}
$$

To analyzes the stability relative to the equilibrium $\left(\begin{array}{ll}x_{1}^{e} & x_{2}^{e^{T}}\end{array}\right)^{T}=\left(\begin{array}{ll}1 & 0^{T}\end{array}\right)^{T}$, consider the following candidate Lyapunov function $V_{1}: \mathbb{S}^{3} \longrightarrow \mathbb{R}$

$$
V_{1}=x_{2}^{T} x_{2}+\left(x_{1}-1\right)^{2}=2\left(1-x_{1}\right)
$$

Since $x_{2}^{T} x_{2}=1-x_{1}^{2} \geqslant 1-x_{1}$, the derivative of $V_{1}$ along the trajectories of (26) with initial condition $\mathbb{S}^{3} \backslash\left(\begin{array}{ll}-1 & 0^{T}\end{array}\right)^{T}$ is given by:

$$
\dot{V}_{1}=-K 1 x_{2}^{T} x_{2} \leqslant-\frac{1}{2} K_{1} V_{1} \leqslant 0 \text { for }\left(x_{1} x_{2}^{T}\right)^{T} \neq\left(x_{1}^{e} x_{2}^{e^{T}}\right)^{T}
$$


Then (26) is exponentially stable. Consider now the following variable:

$$
z=x_{3}+K_{1} x_{2}
$$

Hence the system (19)-(20) can be rewritten as:

$$
\left(\begin{array}{l}
\dot{x}_{1} \\
\dot{x}_{2}
\end{array}\right)=-\frac{1}{2} K_{1} \Xi\left(x_{1}, x_{2}\right) x_{2}+\frac{1}{2} \Xi\left(x_{1}, x_{2}\right) z
$$

The differential equation for $z$ is:

$$
\dot{z}=-J^{-1}\left[x_{3}^{\times}\right] J x_{3}+J^{-1} u+K_{1} \dot{x}_{2}
$$

One wants to find a control $u$ such that the system (29)-(30) is asymptotically stable. For that consider the following candidate Lyapunov function $V: \mathbb{S}^{3} \times \mathbb{R}^{3} \longrightarrow \mathbb{R}$ defined by

$$
V=V_{1}+\frac{1}{2} z^{T} K_{3}^{-1} J z
$$

where $K_{3}=I_{3} k_{3}$ and $k_{1} \in \mathbb{R}^{+}$. The derivative of $V$ along the trajectories of (29)-(30) with initial condition $\mathbb{S}^{3} \times \mathbb{R}^{3} \backslash$ $\left(\begin{array}{lll}-1 & 0^{T} & 0^{T}\end{array}\right)^{T}$ is given by:

$$
\begin{aligned}
\dot{V} & =x_{2}^{T} x_{3}+z^{T} \dot{z} \\
& =x_{2}^{T} x_{3}+z^{T} K_{3}^{-1} J\left(-J^{-1}\left[x_{3}^{\times}\right] J x_{3}+J^{-1} u+K_{1} \dot{x}_{2}\right)
\end{aligned}
$$

Now we can summarize the results of the above procedure in the following proposition.

Proposition 3.1: The function $V: \mathbb{S}^{3} \times \mathbb{R}^{3} \longrightarrow \mathbb{R}$ defined by

$$
V=x_{2}^{T} x_{2}+\left(x_{1}-1\right)^{2}+\frac{1}{2} z^{T} K_{3}^{-1} J z
$$

with $z=x_{3}+K_{1} x_{2}$ is a Control Lyapunov Function (CLF) for the system (22) relative to the equilibrium state $x^{e}=$ $\left(\begin{array}{lll}1 & 0^{T} & 0^{T}\end{array}\right)^{T}$ with the stabilizing control:

$$
u=\left[x_{3}^{\times}\right] J x_{3}-J K_{1} \dot{x}_{2}-K_{2} z-K_{3} x_{2}
$$

where $K_{1}, K_{2}, K_{3} \in \mathbb{R}^{3 \times 3}$ are diagonal positive definite matrices.

Proof: Clearly $V$ is smooth and positive definite. Now, consider the derivative of (33) along the trajectories of the closed-loop system with any initial condition in $\mathbb{S}^{3} \backslash\left(\begin{array}{lll}-1 & 0^{T} & 0^{T}\end{array}\right)^{T}$

$$
\begin{aligned}
\dot{V}(x) & =\frac{\partial V^{T}}{\partial x} f(x)+\frac{\partial V^{T}}{\partial x} g(x) u \\
& =\underbrace{x_{2}^{T} x_{3}+z^{T} K_{3}^{-1}\left(\left[x_{3}^{\times}\right] J x_{3}+J K_{1} \dot{x}_{2}\right)}_{:=a(x)} \\
& +\underbrace{z^{T} K_{3}^{-1}}_{:=b(x)} u \\
& =-z^{T} K_{3}^{-1} K_{2} z-K_{1} x_{2}^{T} x_{2}<0 \text { for } x \neq x^{e}
\end{aligned}
$$

Then this mean that $x_{2}, z \rightarrow 0$. That implies $x_{3} \rightarrow 0$ and due to the quaternion normality condition $x_{1} \rightarrow 1$.

Besides, note that $b(x)=0$ implies $z=0$ i.e. $x_{3}=-K_{1} x_{2}$ as a consequence $a(x)=-K_{1} x_{2}^{T} x_{2}<0$. Thus, the time derivative of $V$ meets the following requirement:

$$
b(x)=0 \Rightarrow a(x)<0 \text { for all } x \neq x^{e}
$$

Then $V$ given by (33) is a Control Lyapunov Function relative to the equilibrium state $x^{e}=\left(\begin{array}{lll}1 & 0^{T} & 0^{T}\end{array}\right)^{T}$.

Corollary 3.2: (Event-triggered attitude stabilization of Flying robots) Consider the Flying robot attitude kinematics and dynamics and the CLF given by (22) and (33), respectively. Then the event-triggered feedback $(k, e)$ defined by (7)-(8) with $\bar{\theta}=x_{2}^{T} x_{2}+\left(x_{1}-1\right)^{2}$ and $\Delta(x)=\operatorname{diag}\left(\delta_{1}(x), \delta_{2}(x), \delta_{3}(x)\right)$ asymptotically stabilizes the Flying robot at $\left(10^{T} 0^{T}\right)^{T}$ with a domain of attraction equal to $\mathbb{S}^{3} \times \mathbb{R}^{3} \backslash\left(-10^{T} 0^{T}\right)^{T}$. Furthermore, the feedback $(k, e)$ is semi-uniformly MSI and smooth on $\mathbb{S}^{3} \times \mathbb{R}^{3} \backslash\left(\begin{array}{lll}1 & 0^{T} & 0^{T}\end{array}\right)^{T}$.

Proof: The proof follows the one of Theorem 2.3.

Remark 3.3: Note that the stability analysis has been carried out considering the asymptotic condition $q_{d}=\left(\begin{array}{ll}1 & 0^{T}\end{array}\right)^{T}$. In the case where the asymptotic condition $q \rightarrow q_{d}$ with $q_{d} \neq\left(10^{T}\right)^{T}$ is considered, the feedback becomes in function of $x_{1}=q_{e_{0}} \in \mathbb{R}, x_{2}=q_{e_{v}} \in \mathbb{R}^{3}$, $x_{3}=\omega \in \mathbb{R}^{3}$, where the $q_{e}$ is given by (17) which represents the attitude error between the current orientation and the desired one.

Remark 3.4: The event-triggered feedback developed here is carried out in a continuous-time framework and it satisfies the Minimal inter-Sampling Interval property (MSI) (see Definition 2.1) to avoid zero inter-sampling time leading to Zeno phenomena. In practical, the digital implementation forces the event function to be monitored periodically, instead that continuously, which guarantees a minimum sampling period (the control can not be updated more often than the a priori given sampling period). The effects of this bound will be addressed in the future.

\section{REAL-TIME APPLICATION}

This section is devoted to proving the effectiveness of the proposed attitude event-triggered control under a real platform. For this, a four-rotor mini helicopter has been developed for the "Dynamical Systems and Control" group of the Autonomous University of Puebla (BUAP), Mexico, see Fig. 3.

\section{A. System model}

The quadrotor is a small aerial vehicle that belongs to the VTOL (Vertical Taking Off and Landing) class of aircrafts. It is lifted and propelled, forward and laterally, by controlling 
the rotational speed of four blades mounted at the four ends of a simple cross and driven by four DC Brushless motors (BLDC). On such a platform (see Fig. 2), given that the front and rear motors rotate counter-clockwise while the other two rotate clockwise, gyroscopic effects and aerodynamic torques tend to cancel each other out in trimmed flight. The rotation of the four rotors generates a vertical force, called the thrust $T$, equal to the sum of the thrusts of each rotor $\left(T=f_{1}+f_{2}+f_{3}+f_{4}\right)$. The pitch movement $\theta$ is obtained by increasing/decreasing the speed of the rear motor while decreasing/increasing the speed of the front motor. The roll movement $\phi$ is obtained similarly using the lateral motors. The yaw movement $\psi$ is obtained by increasing/decreasing the speed of the front and rear motors while decreasing/increasing the speed of the lateral motors. In order to avoid any linear movement of the quadrotor, these maneuvers should be achieved while maintaining a value of the total thrust $T$ that balances the aircraft weight. In order to model the system's dynamics, two frames are defined: a fixed frame in the space $\mathbf{E}^{f}=\left[\vec{e}_{1}^{f}, \vec{e}_{2}^{f}, \vec{e}_{3}^{f}\right]$ and a body-fixed frame $\mathbf{E}^{b}=\left[\vec{e}_{1}^{b}, \vec{e}_{2}^{b}, \vec{e}_{3}^{b}\right]$, attached to the quadrotor at its center of gravity, as shown in Fig. 2.

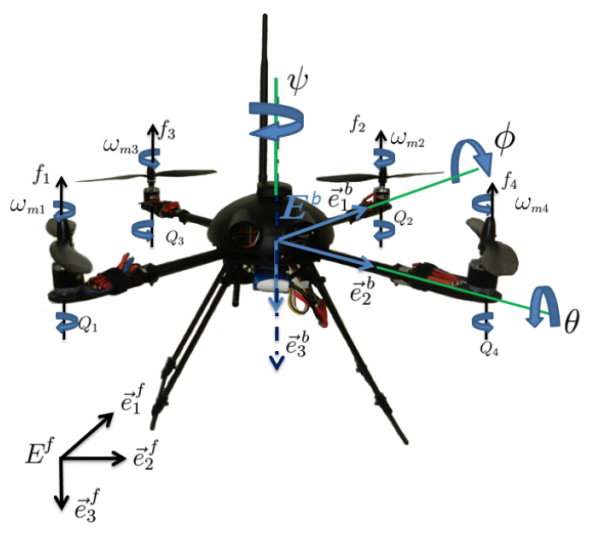

Fig. 2. Quadrotor: fixed frame $\mathbf{E}^{f}=\left[\vec{e}_{1}^{f}, \vec{e}_{2}^{f}, \vec{e}_{3}^{f}\right]$ and body-fixed frame $\mathbf{E}^{b}=\left[\vec{e}_{1}^{b}, \vec{e}_{2}^{b}, \vec{e}_{3}^{b}\right]$

According to [28], [7] and II-B, the six degrees of freedom model (position and attitude) of the system can be separated into translational and rotational motions, represented respectively by $\Sigma_{T}$ and $\Sigma_{R}$ in equation (36) and (37).

$$
\begin{gathered}
\Sigma_{T}:\left\{\begin{array}{l}
\dot{p}=v \\
\dot{v}=g e_{3}-\frac{1}{m_{h}} R^{T}(q) T e_{3}
\end{array}\right. \\
\Sigma_{R}:\left\{\begin{array}{c}
\dot{q}=\frac{1}{2} \Xi(q) \omega \\
J \dot{\omega}=-\left[\omega^{\times}\right] J \omega+\Gamma
\end{array}\right.
\end{gathered}
$$

where $m_{h}$ denotes the mass of the quadrotor and $J$ its inertial matrix expressed in $\mathbf{E}^{b} \cdot g$ is the gravity acceleration and $e_{3}=\left(\begin{array}{lll}0 & 0 & 1\end{array}\right)^{T} \cdot p=\left(\begin{array}{lll}x & y & z\end{array}\right)^{T}$ represents the position of the quadrotor's center of gravity, which coincides with the origin of frame $\mathbf{E}^{b}$, with respect to frame $\mathbf{E}^{f}, v=\left(v_{x} v_{y} v_{z}\right)^{T}$ its linear velocity in $\mathbf{E}^{f}$, and $\omega$ denotes the angular velocity of the quadrotor expressed in $\mathbf{E}^{b} . \Gamma \in \mathbb{R}^{3}$ depend on the couples generated by the actuators, aerodynamic couples and external couples (environmental forces). In this paper, it is assumed that these torques are only generated by the actuators. $-T e_{3}$ is the total thrust expressed in $\mathbf{E}^{b}$.

The reactive torque $Q_{j}$ due to the $j^{\text {th }}$ rotor drag, $j \in$ $\{1,2,3,4\}$, and the total thrust $T$ generated by the four rotors can be approximated by an algebraic relationship on function of a PWM control signal applied to the BLDC-drivers:

$$
Q_{j}=k_{m} u_{m j} \quad T=b_{m} \sum_{j=1}^{4} u_{m j}=\sum_{j=1}^{4} f_{j}
$$

where the input signals $u_{m i}$ are expressed in seconds, i.e. the time during which the PWM control signal is in high state. $k_{m}>0$ and $b_{m}>0$ are two parameters that depend on the air density, the dynamic pressure, the lift coefficient, the radius and the angle of attack of the blades and they are obtained experimentally.

The components of the control torque vector $\Gamma$ generated by the rotors are given by:

$$
\begin{aligned}
& \Gamma_{1}=d b_{m}\left(u_{m 3}-u_{m 4}\right) \\
& \Gamma_{2}=d b_{m}\left(u_{m 1}-u_{m 2}\right) \\
& \Gamma_{3}=k_{m}\left(-u_{m 1}+u_{m 2}-u_{m 3}+u_{m 4}\right)
\end{aligned}
$$

with $d$ being the distance from one rotor to the center of mass of the quadrotor. Combining equations (38) and (39), the forces and torques applied to the quadrotor are written as:

$$
\begin{aligned}
\left(\begin{array}{l}
\Gamma \\
T
\end{array}\right) & =\left(\begin{array}{cccc}
0 & 0 & d b_{m} & -d b_{m} \\
d b_{m} & -d b_{m} & 0 & 0 \\
-k_{m} & k_{m} & -k_{m} & k_{m} \\
b_{m} & b_{m} & b_{m} & b_{m}
\end{array}\right)\left(\begin{array}{c}
u_{m 1} \\
u_{m 2} \\
u_{m 3} \\
u_{m 4}
\end{array}\right) \\
& =N U_{m}
\end{aligned}
$$

where $U_{m}=\left(\begin{array}{llll}u_{m 1} & u_{m 2} & u_{m 3} & u_{m 4}\end{array}\right)^{T}$. Since $N$ is an invertible matrix, the vector of signals control $U_{m}$ is easily obtained.

\section{B. Experimental results}

Considering the only the rotational motion of the quadrotor helicopter represented by $\Sigma_{r}$ in (37). The control law (7)-(8) is executed on a Pipistrello Board which contains a FPGA Spartan-6 LX45-3. The Spartan-6 has the ability to implement a "MicroBlaze" soft processor running at $100 \mathrm{MHz}$. Furthermore, the Spartan-6 has the advantage to develop custom modules such as PWM generators, Digital input-output ports and USARTs ports. An AHRS (Microstrain 3DM-GX3-45) is used to obtain the attitude quaternion and angular velocity at $73 \mathrm{~Hz}$. A Xbee -Pro Module linked to a $\mathrm{PC}$ is used to exchange the processed data. The desired attitude $q_{d}$ is provided by means of a 6-channel Radio-Control Futaba R2006GS with $2.4 \mathrm{GHz}$ radio technology. Four power modules are used to drive the motors by means of a PWM signal. The frequency of 
the PWM signal is fixed to $500 \mathrm{~Hz}$. The power of the whole system is supplied by a 11.1 Volts Li-Po battery. The specification and parameters of the quadrotor prototype are given in the Table I.

\begin{tabular}{|c|c|c|c|}
\hline Parameter & Description & Value & Units \\
\hline$m$ & Mass & 1.490 & $\mathrm{Kg}$ \\
$d$ & Distance & 0.27 & $\mathrm{~m}$ \\
$J_{x}$ & Inertia in X-axis & $34.3 \times 10^{-3}$ & $\mathrm{Kg} \cdot \mathrm{m}^{2}$ \\
$J_{y}$ & Inertia in y-axis & $34.4 \times 10^{-3}$ & $\mathrm{Kg} \cdot \mathrm{m}^{2}$ \\
$J_{z}$ & Inertia in Z-axis & $52.9 \times 10^{-3}$ & $\mathrm{Kg} \cdot \mathrm{m}^{2}$ \\
$b_{m}$ & Proportionality Constant & 8548 & $\mathrm{~N} / \mathrm{s}$ \\
$k_{m}$ & Proportionality Constant & 1898 & $\mathrm{~N} \cdot \mathrm{m} / \mathrm{s}$ \\
\hline
\end{tabular}

TABLE I

PARAMETERS OF THE SYSTEM

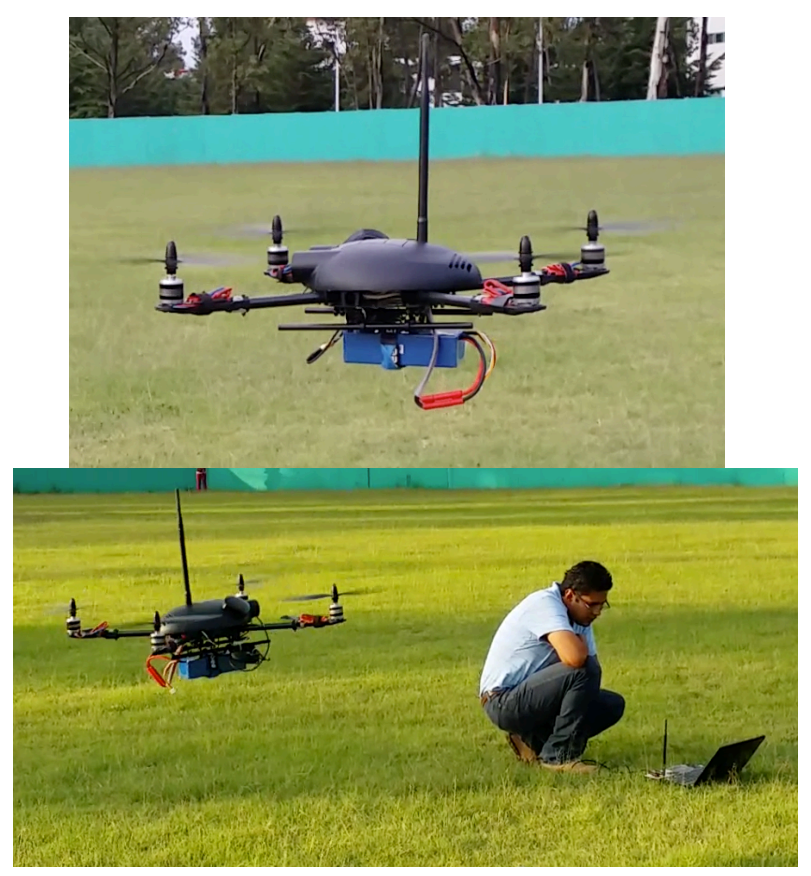

Fig. 3. The quadrotor mini-helicopter in flight

To evaluate the benefices of the control law defined in the corollary 3.2, an experiment is performed. The objective is to bring the quadrotor from any initial orientation, sufficiently far from the desired attitude defined by $q_{d}=\left(\begin{array}{ll}1 & 0^{T}\end{array}\right)^{T}$ i.e. $\phi_{d}=\theta=0=\psi=0$ and hold it there by maintaining the angular velocity to zero. The desired thrust is taken as $T \geq m g=14.63 \mathrm{~N}$ such that it guarantees a balance of the quadrotor's weight. Experiments were performed with the following gains: $K_{1}=\operatorname{diag}(7.2,7.2,3.6)$, $K_{3}=\operatorname{diag}(1.51,1.51,0.151)$ whose are necessary for the determination of $a(x)$ and $b(x)$ (see (35)) used in the control law (7). The value for the parameter $\Delta$ is fixed to $\Delta=\operatorname{diag}(0.25,0.25,0.18)$. These parameters are choosing (in practical) such that one obtains an acceptable stabilization time while satisfying bounds on control signal, in order to avoid actuator saturation.
In the experiment, the control capabilities are tested to stabilize the system, with initial conditions $\left(-20^{\circ}, 20.4^{\circ},-43^{\circ}\right)$. The results are depicted in Fig. 4 where it is showed that the stabilization takes about 2.5 seconds. The first (top) plot shows the Euler angles (since they are more intuitive, however the control law uses quaternions) whereas angular velocities are provided in the second one. The third and fourth plots show the control torques and the Control Lyapunov Function (one can see it decreases while the system is stabilized). Finally, the last (bottom) plots give the event function - an event occurs when this function vanishes to zero, as defined in (3) - and a representation of the call control instants ( 1 and 0 in the last plot mean the control is updated or it is kept constant respectively). In the classical frame (time-triggered control), the control law should be updated 730 times for a span of 10 seconds, since the AHRS continuously provides the state at a frequency of $73 \mathrm{~Hz}$. With the proposed approach, one could note in Fig. 4(f) that some large intervals without any control update exist. Actually, the control law is updated only 126 times during the 10 seconds experimental time, which represents a reduction of $82.7 \%$ w.r.t. the classical frame. It is worth noting that this reduction in the number of updates, reduces the data exchange between AHRS, controller and actuators without sacrificing performance.

\section{CONCLUSIONS}

This paper proposes the development of a nonlinear event-triggered feedback for the attitude stabilization of Flying robots. The attitude is parameterized using the unit quaternion. Firstly, it is proved the existence of a smooth Control Lyapunov Function. Then, an event-triggered static feedback is derived from the universal formula for event-triggered stabilization of general nonlinear systems affine in the control [18] with an event-function based on the control proposed in [19]. The control law ensures the asymptotic stability of the closed-loop system to the desired attitude. The approach is validated in real-time using a quadrotor mini-helicopter. The experiments reveals that the event driven controller reduces by $82.7 \%$ the control update and as a consequence it reduce the communication traffic in the local network without deteriorating the closed-loop system performance.

\section{REFERENCES}

[1] P. Castillo, A. Dzul, and R. Lozano, "Real-time stabilization and tracking of a four-rotor mini rotorcraft," IEEE Trans. on Control Sytems Technology, vol. 12(4), pp. 510-516, 2004.

[2] S. Bouabdallah, A. Noth, and R. Siegwart, "PID vs LQ control techniques applied to an indoor micro quadrotor," in Proc. of the IEEE/RSJ Conference on Intelligent Robots and Systems, vol. 3, 2004, pp. 2451-2456.

[3] S. Bouabdallah and R. Siegwart, "Backstepping and sliding-mode techniques applied to an indoor micro quadrotor," 2005, pp. 2247-2252.

[4] N. Guénard, T. Hamel, and V. Moreau, "Dynamic modelling and intuitive control strategy for an X4-Flyer," in $5^{\text {th }}$ IEEE International Conference on Control and Automation, ICCA'05, vol. 1, Budapest, Hungary, 2005, pp. 141-146. 


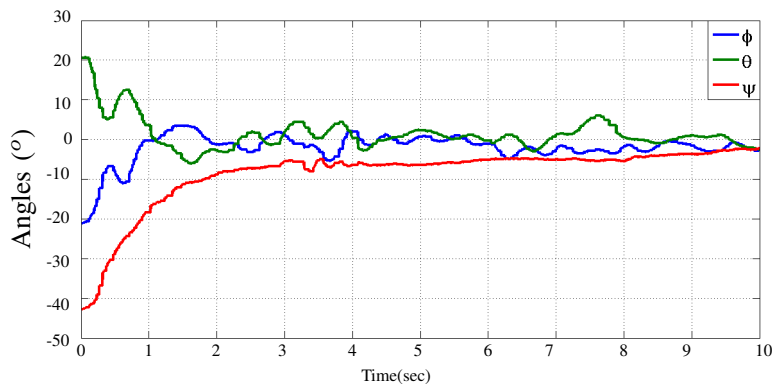

(a) Quadrotor attitude

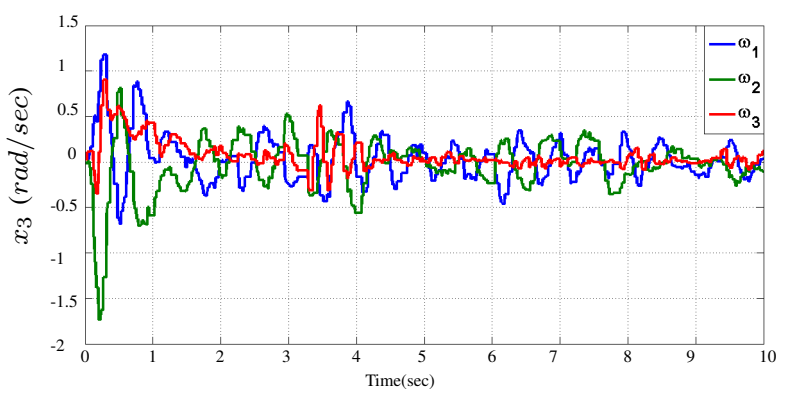

(b) Angular velocities

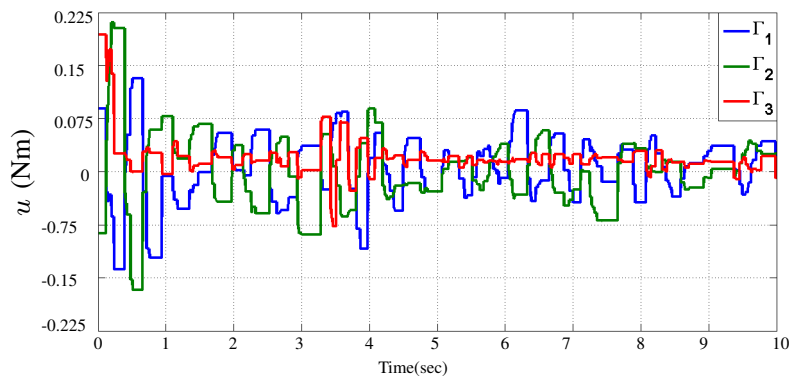

(c) Control

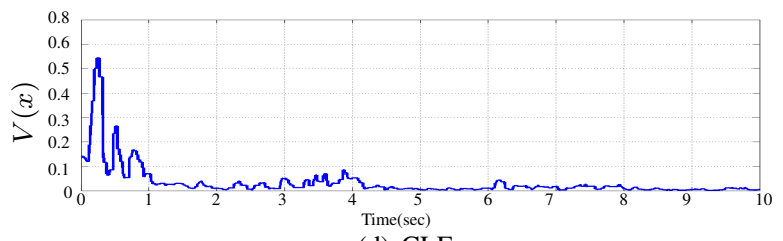

(d) CLF

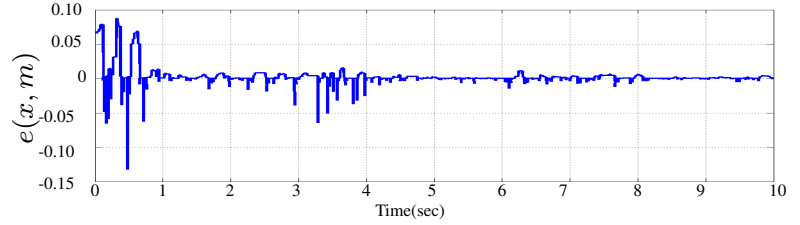

(e) Event function

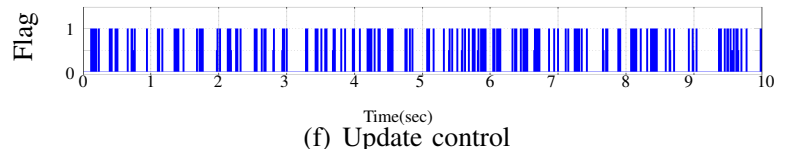

(f) Update control

Fig. 4. Stabilization to the origin of the quadrotor.

[5] A. Tayebi and S. McGilvray, "Attitude stabilization of a VTOL quadrotor aircraft," IEEE Transactions on Control Systems Technology, vol. 14 , no. 3, pp. 562-571, 2006.

[6] P. Castillo, P. Albertos, P. Garcia-Gil, and R. Lozano, "Simple real-time attitude stabilization of a quad-rotor aircraft with bounded signals," in 45th IEEE Conference on Decision and Control, San
Diego, CA, USA, 2006, pp. 1533-1538.

[7] J. Guerrero-Castellanos, N. Marchand, A. Hably, S. Lesecq, and J. Delamare, "Bounded attitude control of rigid bodies: Real-time experimentation to a quadrotor mini-helicopter," Control Engineering Practice, vol. 19, no. 8, pp. 790-797, 2011.

[8] J. J. Corona-Sánchez and H. Rodríguez-Cortés, "Trajectory tracking control for a rotary wing vehicle powered by four rotors," Journal of Intelligent and Robotic Systems, vol. 70, no. 1-4, pp. 39-50, 2013.

[9] K.-E. Årzén, "A simple event-based PID controller," in Preprints of the 14th World Congress of IFAC, Beijing, P.R. China, 1999.

[10] J. Sandee, W. Heemels, and P. van den Bosch, "Event-driven control as an opportunity in the multidisciplinary development of embedded controllers," in Proc. of the IEEE American Control Conference (ACC), 2005, pp. 1776-1781.

[11] S. Durand and N. Marchand, "Further results on event-based PID controller," in Proc. of the European Control Conference (ECC), 2009.

[12] J. Sánchez, M. Guarnes, S. Dormido, and A. Visioli, "Comparative study of event-based control strategies: An experimental approach on a simple tank," in Proc. of the European Control Conference (ECC), 2009.

[13] W. Heemels, J. Sandee, and P. van den Bosch, "Analysis of event-driven controllers for linear systems," International journal of control, vol. 81, pp. 571-590, 2009.

[14] J. Lunze and D. Lehmann, "A state-feedback approach to event-based control," Automatica, vol. 46, pp. 211-215, 2010.

[15] K. Åström and B. Bernhardsson, "Comparison of Riemann and Lebesque sampling for first order stochastic systems," in Proc. of the IEEE Conference on Decision and Control (CDC), 2002.

[16] M. Velasco, P. Martí, and E. Bini, "On Lyapunov sampling for event-driven controllers," in Proc. of the IEEE Conference on Decision and Control (CDC), 2009.

[17] P. Tabuada, "Event-triggered real-time scheduling of stabilizing control tasks," IEEE Trans. on Automatic Control, vol. 52, no. 9, pp 1680-1685, 2007.

[18] N. Marchand, S. Durand, and J. F. Guerrero-Castellanos, "A general formula for event-based stabilization of nonlinear systems," IEEE Trans. on Automatic Control, vol. 58, no. 5, 2013.

[19] N. Marchand, J. Martinez, S. Durand, and J. F. Guerrero-Castellanos, "Lyapunov event-triggered control: a new event strategy based on the control," in Proc. of the 9th IFAC Symposium on Nonlinear Control Systems (NOLCOS), 2013, hal-00828391.

[20] M. Lemmon, "Event-triggered feedback in control, estimation, and optimization," in Networked Control Systems Lecture Notes, ser. Control and Information Sciences Networked Control Systems, A. Bemporad, M. Heemels, and M. Johansson, Eds. Springer-Verlag, 2010, vol. 405, pp. 293-358.

[21] J. Téllez-Guzmán, J. Guerrero-Castellanos, S. Durand, N. Marchand, and L. Maya, "Event-based LQR control for attitude stabilization of a quadrotor," in Proc. of the IFAC Latinoamerican Control Conference (CLCA), 2012, pp. 1-6.

[22] E. D. Sontag, "Clocks and insensitivity to small measurement errors," ESAIM Control, Optimisation and Calculus of Variations, vol. 4, pp. 537-557, 1999.

[23] _ Mathematical control theory, deterministic finite dimensional systems, 2nd ed. New York Berlin Heidelberg: Springler Verlag, 1998

[24] M. D. Shuster, "A survey of attitude representations," Journal of the astronautical sciences, vol. 41, no. 4, pp. 439-517, 1993.

[25] R. Schlanbusch, A. Loria, and P. J. Nicklasson, "On the stability and stabilization of quaternion equilibria of rigid bodies," Automatica, vol. 48, no. 12, pp. 3135 - 3141, 2012. [Online]. Available: http://www.sciencedirect.com/science/article/pii/S000510981200413X

[26] S. Bhat and D. Bernstein, "A topological obstruction to global asymptotic stabilization of rotational motion and the unwinding phenomenon," Systems \& Control Letters, vol. 39, pp. 63-70, 2000.

[27] N. A. Chaturvedi, M. K. Sanyal, and N. H. McClamroch, "Rigid-body attitude control," IEEE Control Systems Magazine, vol. 31, no. 3, pp. 30-51, June 2011.

[28] P. Pounds, R. Mahony, P. Hynes, and J. Roberts, "Design of a four-rotor aerial robot," in Australian Conference on Robotics and Automation, 2002. 